\title{
REFLEXIÓN SOCIOMORAL Y JUVENTUD DEL CANTÓN DE LOS CHILES: CONSIDERACIONES ACERCA DE LA CALIDAD EDUCATIVA RURAL ${ }^{1}$
}

\author{
SOCIOMORAL REFLECTION AND YOUTH FROM LOS CHILES: \\ ISSUES ABOUT RURAL QUALITY EDUCATION
}

Natacha Monestel Mora*

RESUMEN

Esta investigación intenta un acercamiento a la reflexión sociomoral de adolescentes escolarizados que se han desarrollado en Los Chiles, zona rural costarricense. Con ese fin se realizó un estudio cuantitativo que puso en perspectiva la realidad de 210 jóvenes rurales, en relación con un grupo de adolecentes urbanos entre 14 y 22 años. Se trata de abrir la discusión acerca de las diferencias en el acceso a una educación de calidad y sus posibles influencias en la subjetividad de los actores sociales que se desarrollan en dichos contextos.

PALABRAS CLAVE: ADOLESCENTES * CONDICIONES SOCIALES * SISTEMA EDUCATIVO * JUVENTUD RURAL * RAZONAMIENTO MORAL

\section{ABSTRACT}

This research attempts an approach to sociomoral reflection of schooled adolescents from Los Chiles, Costa Rican rural zone. For that purpose, a quantitative study was conducted to set in perspective the reality of 210 rural youngsters, in relation to a group of urban adolescents between 14 and 22 years old. The objective is to open the discussion about the difference in the access to quality education and its influences in the subjectivity of the social actors from these contexts.

KEYWORDS: ADOLESCENTS * SOCIAL CONDITIONS * EDUCATION SYSTEM * RURAL YOUTH * MORAL REASONING

1 En este artículo es el resultado del proyecto de investigación "Desarrollo sociomoral y estilos de apego en adolescentes del cantón de Los Chiles" (Monestel, 2011).

* Instituto Interamericano de Derechos Humanos (IIDH) de Costa Rica. nmonestel@gmail.com 


\section{INTRODUCCIÓN}

Es innegable que actualmente, justo al principio de la segunda década del siglo xxi, las esferas socioeconómicas fundadas a partir de la colonización, han sido transformadas por la globalización. Estas estructuras sociales se caracterizaron por la orientación hacia el trabajo en el campo y la familia extensa, en el caso de la sociedad rural y la orientación reflejada por la familia nuclear y el trabajo remunerado, como base de las sociedades que se desarrollaron en los contextos más urbanos (Aguilar, Angulo, Cerdas, Céspedes, Monge, Ovares, Solórzano y Van Kampen, 2003).

Estas aceleradas transformaciones han afectado de manera distinta las estructuras socioeconómicas durante las últimas tres décadas. Es así como, surge la necesidad de plantear el enfoque de "nueva ruralidad", cuyo fin involucra proponer un desarrollo rural sostenible a partir del análisis de los fenómenos que afectan los espacios rurales (Diez, 2001; Piñeiro, 2001; Teubal, 2001; Echeverri y Ribero, 2002; De Grammont, 2008).

A través de este artículo, se intenta una aproximación a la nueva ruralidad definida desde los parámetros expuestos, partiendo del estudio de las estructuras de pensamiento moral de adolescentes escolarizados en el cantón de Los Chiles. Además, con el fin de brindar validez interna al estudio y mostrar índices con valores comparables en relación con el comportamiento de las variables en el contexto de la nueva ruralidad costarricense, se considera un grupo de adolescentes que residen en un cantón urbano, quienes son estudiantes de un colegio del cantón de Desamparados. Se trata de evidenciar que, si bien el sistema educativo intenta ofrecer una cobertura en todo el país, en términos de calidad educativa hace falta considerar las especificidades que dependen del entorno, en cuanto a las distintas formas de vivir la juventud.

Es importante mencionar que se parte de la premisa de que el sujeto se desarrolla como actor social influenciado por el contexto $y$ a su vez, transformador de dicho entorno. De ahí que resulta imprescindible abordar las condiciones y las implicaciones de su comportamiento, poniendo atención a los aspectos psicosociales que intervienen en el cambio de su ambiente cotidiano (Alvarado, Ospina-Alvarado y García, 2012).

Siguiendo con esta línea de reflexión, la literatura de Teubal (2001) y Tapia, Castro y Monestel (2007) plantea la necesidad del estudio del desarrollo sociomoral para la comprensión de las adolescencias y su relación con los cambios que ocurren durante esta etapa del ciclo vital, sobre todo cuando se consideran contextos que muestran indicadores con desventajas sociodemográficas, ya que dicho cambio suele ser más dinámico y causante de vulnerabilidad social (Krauscopf, 2007 y Tapia, Castro y Monestel, 2007). Por esta razón, cabe preguntarse por este proceso cuando ocurre en un entorno que enfrenta las aceleradas transiciones sociales, políticas y económicas derivadas de la globalización en condiciones desventajosas (Teubal, 2001; Krauscopf, 2007 y Tapia, Castro y Monestel, 2007).

En este sentido, Los Chiles es el cantón con más bajó índice de desarrollo social; las y los jóvenes que asisten a colegios públicos en esta zona tienen un futuro profesional incierto $y$ sus oportunidades laborales son escasas (INEc, 2007). Asimismo, la expulsión del sistema educativo se disfraza de deserción escolar en esta zona, por lo que la emigración se presenta como una "opción" poco viable (Teubal, 2001). De ahí que se propone incentivar aquellas políticas públicas educativas que fomenten el pensamiento crítico, que impulsen la reflexión acerca del orden establecido y por ende, que sus actores sociales sean los que propongan la transformación de su espacio sin perder de vista su idiosincrasia.

\section{CONTEXTOS RURALES COSTARRICENSES}

En la segunda mitad del siglo xix, en los países de la región centroamericana, ocurrieron cambios en la estructura socioeconómica $y$ política, basados en modelos de sociedades europeas. Surge en esta época, el trabajo en las fábricas e industrias, provocando una separación más marcada entre la cultura de clases altas y populares, así como, una división entre la cultura urbana y la rural (Aguilar et ál., 2003). 
Las caracterizaciones tradicionales del mundo rural han tendido a definirlo a partir de su modo de producción agropecuario, así como por su falta de acceso a los servicios básicos (agua, luz, teléfono y vías de acceso). Sin embargo, las transformaciones significativas que han tenido estos contextos, han provocado que sus sociedades agrarias, organizadas en torno a la actividad primaria, se configuren como mundos rurales más diversificados, de forma tal que su población se integra cada vez más a las actividades laborales vinculadas con el medio urbano, tales como el comercio y los servicios (Pérez, 2001 y De Grammont, 2008).

Por las razones expuestas, es preciso definir lo rural como aquellos espacios que han tenido una historia específica, a partir de la explotación de los recursos naturales de un territorio común para todos sus actores sociales. De esta forma, en estos contextos surgen determinadas relaciones socioculturales, económicas y tecnológicas que vinculan a sus habitantes entre sí, con su ambiente natural y con otras actividades no agrícolas (Sepúlveda y Zuñiga, 2008).

Desde esta perspectiva, se definen los espacios rurales como territorios en donde se localizan uno o más grupos humanos articulados entre sí. Se pueden identificar allí unidades productivas de distintos tamaños, formas específicas de división del trabajo y cadenas de producción y consumo. Por lo tanto, resulta fundamental considerar sus organizaciones sociopolíticas, para de esta forma reconocer los intereses de sus habitantes (Miranda y Matos, 2002). Así, identificar dichas variables, permite comprender mejor los diversos grupos sociales que conforman el territorio rural, entendiéndolo como una micro-región con una identidad natural y cultural única (Miranda y Matos, 2002).

Asimismo, es posible encontrar diferentes criterios operativos para delimitar territorios según sean los objetivos de política de los estados, por ejemplo: físico-naturales (cuencas hidrográficas, áreas ambientalmente protegidas o agroecosistemas específicos); o bien, niveles de gobierno político-territoriales (el departamento, la provincia o el municipio) (Llambí y Pérez, 2007).
En este artículo se considera el cantón de Los Chiles como unidad de análisis, contemplando que dicho territorio fronterizo forma parte de la zona norte del país, la cual comprende una región vinculada a partir de la cuenca del Río San Juan. Es importante recalcar que, según Llambí y Pérez "el territorio, además de una categoría analítica u operativa, es también una construcción social según la perspectiva normativa o cognitiva de quienes en él habitan y de sus poblaciones vecinas" (2007: 54).

Por lo tanto, se entiende que Los Chiles no es solo un espacio físico, sino que en este se desarrollan un conjunto de relaciones sociales. De dichos vínculos, se origina y se expresa una identidad, que corresponde a los objetivos comunes de sus habitantes, así como de sus organizaciones públicas y privadas, de tal forma que se da una construcción social específica. Así, su ubicación ha determinado sus características sociodemográficas, económicas y culturales, ya que los vínculos establecidos a partir de la red fluvial, han intercomunicado a los habitantes de Costa Rica con Nicaragua y las actividades comerciales han dependido, en mayor parte, de este medio de comunicación (Instituto Interamericano de Cooperación para la Agricultura, 2007).

Este territorio estuvo relativamente aislado de los cascos urbanos de ambos países hasta la década de 1970, cuando el acondicionamiento y mejoras de la red vial permitieron vincular esta zona con los centros urbanos más cercanos. Desde entonces, las actividades económicas se han ido transformando $y$ durante los últimos años estos cambios han sido exponenciales (IICA, 2007).

En este contexto, la agroindustria ha tenido un impacto trascendental, transformando los modelos de subsistencia tradicionales $y$ dando paso al surgimiento de las relaciones laborales asalariadas (Piñeiro, 2001). Esto tiene una profunda incidencia en los patrones de vida de la población, generando transformaciones en el imaginario social, las cuales se manifiestan a partir de cambios en los intereses, valores, expectativas, costumbres $y$ actividades cotidianas, tales como: hábitos alimenticios, gustos 
musicales, formas de vestir e interactuar, formas de organizar el trabajo, etc. (Diez, 2001; Aguilar et ál., 2003). El impacto social de dichos cambios es complejo $y$ en muchos casos negativo, ya que los esquemas de valores de las sociedades urbanas y rurales responden a procesos sociohistóricos diferentes.

Existen indicadores que muestran que el bienestar privado y la calidad de los servicios públicos de los espacios rurales, han descendido en relación con años anteriores. Son característicos los altos índices de desempleo, los bajos salarios, la falta de diversidad de oportunidades laborales y se reportaron mayores registros de analfabetismo y deserción escolar (Pujol, 2006). Además, el acceso a la tierra continúa siendo un problema crítico, ya que los coeficientes de concentración o latifundismo se mantienen muy altos (Miranda y Matos, 2002).

Lo anterior podría encontrar una explicación en el fracaso de los proyectos de desarrollo rural y la ausencia de políticas públicas localizadas. Durante la segunda mitad del siglo $\mathrm{xx}$, predominaron las intervenciones neoliberales de desarrollo, las cuales plantean estrategias que se orientan a la apertura económica y a la no intervención pública, como si fueran recetas aplicables a todos los contextos, a pesar de sus claras diferencias sociohistóricas y culturales. Se sostiene que la mayoría de los gobiernos abandonaron las políticas sectoriales enfocadas en el desarrollo rural $y$ se concentraron en las políticas macroeconómicas (Trpin, 2005).

Ante este panorama, surge el enfoque de desarrollo rural sostenible o "nueva ruralidad" (Aguilar et ál., 2003). Esta perspectiva enfatiza ampliar la visión de lo rural más allá de lo agrario y adoptar una perspectiva multidimensional para comprender dichos espacios (Miranda y Matos, 2002; Acosta, 2008). Se trata de abordar la intensa interrelación entre lo urbano y lo rural, lo global y lo local, y los cambios en los patrones culturales de sus habitantes (Miranda y Matos, 2002).

Las propuestas de acción se enfocan en las capacidades de los sujetos para replantear sus propias estrategias de vida (Kay, 2007). Es así como la metodología sugerida se ha orientado a partir de principios de educación popular $y$ de planeación participativa, haciendo protagonista a la población en la identificación y la gestión de iniciativas (Miranda y Matos, 2002). Por esta razón, son fundamentales las políticas públicas educativas que promuevan programas curriculares y extracurriculares orientados a desarrollar capacidades, actitudes y pensamiento crítico en los sujetos, de tal forma que les permita ejercer su ciudadanía.

En este punto, conviene rescatar la noción de ciudadanía planteada por Adela Cortina (2006), quien sugiere que este concepto se refiere a la búsqueda del bien común, a partir del reconocimiento de los derechos y los deberes de los ciudadanos y ciudadanas de una comunidad política. Se entiende que la noción de ciudadanía considera los valores de justicia, solidaridad e igualdad como pilares para el logro del bienestar de una sociedad. El ciudadano o ciudadana, por tanto, es aquel sujeto de derechos civiles, políticos, sociales, culturales, económicos y ambientales. De esta forma, ejercer la ciudadanía implica desde tomar decisiones con respecto a la representación política y las formas de producción y consumo, hasta garantizar el respeto y consideración por el medio ambiente y las distintas culturas que habitan en la comunidad política (Cortina, 2006).

Considerando estas nociones sobre ciudadanía y bienestar común, se sugieren como estrategia de intervención, planes que incluyan la cultura en su sentido socioantropológico, como un factor fundamental en la delimitación de los territorios. De tal forma, que dicho dimensionamiento contribuya a una mejor comprensión de las distintas formas de organización social, que favorezca el mapeo de las redes de solidaridad establecidas y que fomente la participación popular en las comunidades (Miranda y Matos, 2002).

Se trata de lograr una mejor comprensión de las identidades culturales y sus diferentes formas de manifestación. Esto contribuye a revelar la identidad y la voluntad de un colectivo, lo cual muchas veces coexiste en contradicción con los elementos económicos neoliberales. Enfatizar en los factores históricos, culturales, ambientales e institucionales, contribuye a una comprensión integral de las 
especificidades territoriales, las cuales se manifiestan en diferentes formas de apropiación de los recursos naturales, sus productos y su idiosincrasia (Miranda y Matos, 2002).

En definitiva, el enfoque de desarrollo rural sostenible involucra necesariamente la discusión de valores éticos y sociales. Se trata de reflexionar acerca de la solidaridad intergeneracional e interregional, así como de considerar las relaciones sustentables con el medio ambiente. Abrir el debate sobre los valores dominantes en las distintas comunidades posibilita el cambio de aquellos que son incompatibles con la sostenibilidad (Miranda y Matos, 2002; Trpin, 2005 y Kay, 2007).

Expuesto lo anterior y centrando el tema en la discusión que se abordará en este artículo, vale la pena preguntarse si las políticas públicas educativas están contribuyendo a fomentar valores $y$ actitudes que promuevan el pensamiento crítico, de forma tal que la educación pública dote a sus actores sociales de herramientas para analizar y proponer iniciativas de cambio desde su propia realidad rural.

\section{ADOLESCENCIA RURAL ESCOLARIZADA Y DESARROLLO DEL RAZONAMIENTO SOCIOMORAL}

Para abordar el concepto de adolescencia, es importante traer a colación la discusión que plantea Dina Krauscopf (2007) acerca de esta etapa del desarrollo. De acuerdo con esta autora, durante este período se intensifican las interacciones entre los cambios psicosexuales del individuo y las metas socialmente disponibles. Por lo tanto, no se puede considerar como un fenómeno inmutable, sino que debe comprenderse como el resultado de las condiciones histórico-sociales. Una delimitación de esta etapa puede concebirse marcando su inicio con los cambios biológicos y finalizando con la asunción de derechos y deberes sexuales, económicos y legales (Krauscopf, 2007).

Desde esta perspectiva, se considera que hay distintas maneras de ser joven. Se puede decir que las juventudes responden a las características del entramado social en el cual se desarrollan y que varían según sean sus condiciones de clase social, cultura, espacio geográfico donde habitan y la generación a la que pertenecen (Margulis, 2001). Por esta razón, este enfoque se aleja de aquellas definiciones de adolescencia que no incluyen aspectos de construcción sociocultural.

De ahí que se sugiere hablar de "juventudes" para contemplar la pluralidad que caracteriza a los grupos de jóvenes, de acuerdo a su contexto social inmediato. Asimismo, se podría utilizar el concepto de "juventudes rurales", ya que en términos sociales y culturales, la juventud se construye alrededor de un espacio geográfico o una región con sus especificidades idiosincráticas (Portilla y Barrantes, 2003).

La desigualdad de oportunidades de educación, empleo y seguridad social son factores que de acuerdo a la definición planteada, determinarán las diferentes formas de vivir la juventud. Esto se ve reflejado en las inequidades que sufren las y los jóvenes empobrecidos que viven en zonas marginales urbanas o rurales (Donas, 2001), en donde la deserción o expulsión del sistema educativo y la baja calidad de la educación, son algunas de las problemáticas que obstaculizan su desarrollo.

Cabe mencionar que según Díaz-Palacios (2013), los parámetros para medir la calidad educativa deben ser planteados a partir de la transformación que ha caracterizado las nuevas formas de acceso a la información y construcción del conocimiento. Además, se debe considerar hasta qué punto el sistema educativo cumple con lo establecido en las políticas públicas, de tal forma que se evalúe el logro, en cuanto a contenido y diseño curricular, en todas las latitudes $y$ alcance de dicho sistema. Estos alcances deberían suscitar la satisfacción de las necesidades de la comunidad, contribuyendo a mejorar $y$ transformar el nivel de vida de sus actores sociales en forma equitativa, solidaria y justa.

De este modo, fomentar el pensamiento crítico a partir de una propuesta curricular planteada desde un enfoque de derechos, se considera como uno de los parámetros fundamentales en la evaluación de una educación de calidad (Instituto Interamericano de Derechos Humanos, 2006). El objetivo gira en torno a proponer iniciativas que contribuyan con la educación de 
sujetos de derechos, capaces de reflexionar acerca de las necesidades de su entorno, además de los recursos y las oportunidades para mejorar su calidad de vida (Cortina, 2003).

Considerando este enfoque, se puede decir que sí existen limitaciones con respecto a la calidad educativa en estas zonas y se incrementan las desventajas sociales, debido a las escasas oportunidades de empleo. Las y los adolescentes rurales se desarrollan en condiciones de desventaja y exclusión, las cuales restringen sus posibilidades inmediatas de superación y aportes a la comunidad (Donas, 2001).

$\mathrm{Al}$ respecto, se debe tomar en cuenta el papel de la escuela en el desarrollo interpsicológico como agente de socialización en un contexto cultural determinado (Vygotsky, 1997), como es el caso del catón de Los Chiles. Es decir, es importante considerar el rol que tiene el sistema educativo en el proceso, a través del cual las y los miembros de una sociedad se apropian de los principios, normas, valores y patrones de comportamiento de su grupo social y cultural (Alvarado, Ospina-Alvarado y García, 2012).

Se propone que la experiencia en la escuela y la alfabetización promuevan un intercambio social basado en herramientas psicológicas o bien, se podría decir que impulsa el desarrollo que va desde las operaciones concretas hasta el razonamiento abstracto (Wertsch, 1988; Cole y Engeström, 1993; Vygotsky, 1997). En otras palabras y siguiendo a Piaget (1991), el pasaje del pensamiento concreto al pensamiento abstracto se logra identificar en el momento en el que las niñas $y$ los niños empiezan a reflexionar sobre categorías, símbolos, signos o conceptos que representan su realidad. Mientras que las estructuras de pensamiento concreto son aquellas operaciones mentales referidas a los objetos o procesos tangibles del medio que rodea al niño o la niña durante la primera infancia. Es decir, cuando se da una reflexión en presencia de lo perceptible $y$ aún no se evidencia una abstracción de dicha realidad.

Esta perspectiva teórica/metodológica comprende el razonamiento moral como estructuras de pensamiento que van desde el respeto unilateral a la autoridad (pensamiento concreto), pasando por una asimilación de las convenciones sociales, hasta alcanzar una compresión del orden social que permite el cuestionamiento y análisis de las normas (pensamiento abstracto).

El desarrollo de dicho razonamiento implica que los individuos conceptualicen sus nociones éticas, a partir de su experiencia con el medio social, basándose en sus propias construcciones sobre justicia, equidad, derechos y bienestar de las personas. Así, la reflexión sociomoral se comienza a estructurar de manera sistemática en la infancia y se va transformando durante el proceso de desarrollo, de tal forma que se configuran según sean las condiciones socioambientales de los sujetos (Kohlbelrg, 1992 y Turiel, 2006).

Por las razones expuestas, es importante recalcar el rol de la escuela en el desarrollo del pensamiento sociomoral, ya que esta contribuye con el impulso de las estructuras sociomorales que van desde el razonamiento más concreto (moral preconvencional), pasando por una asimilación de las normas y las convenciones sociales (moral convencional), hasta llegar al análisis de nociones o conceptos más abstractos como la justicia o la equidad (moral postconvencional).

Se desprende de lo anterior, la existencia de una correlación entre el desarrollo del razonamiento moral y el desarrollo del pensamiento conceptual. No obstante, Kohlberg (1992) supone que dicha correlación consiste en que las etapas del desarrollo intelectual serían condiciones necesarias, pero no suficientes para alcanzar una etapa moral análoga. De esta forma, al hablar de desarrollo moral no se puede limitar su compresión al desarrollo de razonamiento cognitivo, ya que este fenómeno involucra a su vez pensamientos, emociones $y$ conductas que se relacionan entre sí y se manifiestan en el medio social.

Por lo tanto, este texto se centra en la dimensión cognoscitiva del desarrollo moral de las y los adolescentes que residen en Los Chiles, ya que interesa el debate sobre dicho aspecto de la vida social de la juventud escolarizada de este cantón, como un primer acercamiento a la compresión de su socialización. Factores como la pobreza, la segregación, las brechas en los 
logros educativos, el trabajo infantil, la migración forzada y la desigual distribución de recursos son cuestiones que deben ser discutidas desde el enfoque planteado, ya que implican manifestaciones opuestas a la justicia, el bienestar, la equidad, los derechos humanos y la participación democrática.

Se trata de abrir la discusión acerca de las diferentes formas de ser joven y poner en perspectiva cómo a través de diferentes naciones y culturas, son evidentes manifestaciones de indignación, resentimiento y contradicción con las normas, especialmente entre grupos en posiciones de poder y subordinados. Este es el caso de las tensiones entre grupos étnicos, clases sociales y géneros (Turiel, 2006). Por esta razón, cabe hacerse la pregunta sobre el razonamiento sociomoral de aquellos grupos en desventaja social, quienes se desarrollan en condiciones injustas y la adversidad del contexto en el que crecen, implica una contradicción con nociones como la equidad, la dignidad, la justicia y la participación ciudadana, entre otras.

\section{METODOLOGÍA \\ a. PARTICIPANTES}

La población de interés fueron las y los adolescentes que estuvieran cursando noveno, décimo y undécimo año en centros educativos del cantón de Los Chiles, ya que se parte del supuesto que correlaciona positivamente el nivel educativo con el desarrollo del razonamiento moral (dimensión cognitiva). De acuerdo al avance a través del sistema educativo, los estadios más maduros del razonamiento moral postconvencional permitirán una reflexión más abstracta $y$ menos concreta, lo cual promueve el análisis crítico de la realidad.

De esta forma, se infiere a partir de la literatura expuesta en este trabajo que, si el sistema educativo fomenta el desarrollo del pensamiento crítico, las y los adolescentes que estén cursando el último ciclo de educación diversificada deberían revelar una mayor tendencia a evidenciar rasgos característicos de los estadios maduros del razonamiento moral (Eisenberg, Spinrad y Sadovsky, 2006; Gibbs, Basinger y Fuller, 1992; Kohlberg, 1992 y Tapia, Castro y Monestel,
2007), los cuales se presentan como las variables de interés en este estudio.

Se consideró trabajar con las y los adolescentes que asistían a los centros educativos con mayor porcentaje de matrícula de dicho cantón, debido a la gran concentración de estudiantes que presentan estas instituciones. La información acerca de la totalidad de estudiantes matriculados se determinó a partir de una base de datos elaborada por el Departamento de Estadística del Ministerio de Educación Pública. Asimismo, se trabajó con un grupo del mismo tamaño de adolescentes del cantón de Desamparados, con el fin de brindar validez interna al estudio.

El cantón de Desamparados se eligió porque presenta una de las mayores densidades poblacionales del país, en donde se calcula que habitan 818 personas por $\mathrm{Km}^{2}$ (Instituto Nacional de Estadística y Censos, 2007). Asimismo, se consideraron otros rasgos tales como el acceso a los medios de comunicación y los servicios. Por otro lado, se puede decir que las $y$ los jóvenes que han crecido en este contexto, se han desarrollado en un ambiente en donde la globalización ha implicado una transformación heterogénea con respecto a la utilización de los recursos naturales y el modo de producción, en general. Las relaciones asalariadas han sido parte de su historia por más de tres generaciones y las posibles desventajas derivadas de las transformaciones del mundo globalizado plantean retos diferentes, tales como la pobreza y su relación con el hacinamiento y los tipos de violencia social que surgen en los escenarios más urbanos (Carrión, 2001 y Reguillo, 1995).

El tamaño de la muestra y la proporción de estudiantes se calculó a partir de un análisis de poder estadístico. De acuerdo con los datos proporcionados por el Departamento de Estadística del Ministerio de Educación Pública, durante el 2008, la totalidad de adolescentes escolarizados entre noveno y undécimo año en los colegios de Pavón y Técnico de Los Chiles es de 378. Por lo tanto, contemplando esta población y basándose en la prueba de poder estadístico realizada a través del programa GPower, se puede establecer que para un estudio en el que se realiza una comparación entre dos grupos independientes, si se 
quiere alcanzar un nivel de significancia de 5\% $(0,05)$, un efecto de correlación $(\mathrm{r})$ moderado de 0,3 y una probabilidad de evitar un error tipo II (1- $\beta$ ) de 0,95 , se sugiere considerar al menos 134 personas para cumplir con los criterios de representatividad (Clark-Carter, 2004). De esta forma, en el estudio principal se trabajó con 155 adolescentes de dichas instituciones y se comparó con un grupo del mismo tamaño de estudiantes de Desamparados (Monestel, 2011: 53).

El territorio rural se eligió en función de sus características sociodemográficas, tales como su baja densidad de población, 14,7 habitantes por $\mathrm{Km}^{2}$ (INEc, 2007). Además, para el estudio de la nueva ruralidad, el análisis de este territorio es pertinente, ya que resaltan las características relacionadas con su ubicación fronteriza. El histórico aislamiento con respecto a los cascos urbanos, debido a las deficientes vías de comunicación, es un factor que ha caracterizado estos espacios, así como, el analfabetismo relacionado con las vulnerabilidades que presentan las poblaciones migrantes debido a las limitaciones de acceso al sistema educativo (Canales, Martínez, Reboiras y Rivera, 2010; IICA, 2007).

Esta zona se ha caracterizado por un crecimiento poblacional de hasta $71,6 \%$ para el año 2006 (IICA, 2007), lo cual se relaciona con las mejoras de la red vial a partir de 1973 y la constante migración proveniente de Nicaragua, de tal forma que para el 2006, se registraron 20000 habitantes en el cantón (IICA, 2007). Los aspectos mencionados provocan que los rasgos de la nueva ruralidad se evidencian en el territorio. En efecto, ha desaparecido la drástica dicotomía entre el campo y la ciudad, gracias al acondicionamiento de la red vial $y$ al acceso a los servicios y medios de comunicación.

Por otro lado, la riqueza de sus suelos ha provocado que históricamente su desarrollo económico se centrara en la agricultura. El cultivo de frutas y caña de azúcar ha sido característico de esta zona. Para finales de la década de 1980, el respaldo gubernamental a los pequeños productores disminuyó considerablemente, favoreciendo así el desarrollo de la agroindustria y la producción de naranja, principalmente (IICA, 2007). Desde entonces, surgen en esta zona, las relaciones laborales asalariadas.

Estos cambios en la economía han traído consigo daños ambientales y estragos en el nivel del desarrollo social del cantón: contaminación agroquímica, desempleo, deserción escolar y conflictos agrarios, son algunos de los problemas asociados al desarrollo desmedido de la agroindustria. Por consiguiente, este cambio en los patrones de vida de la población ha influido en los intereses, valores, expectativas, costumbres y actividades cotidianas (Diez, 2001, Aguilar et ál., 2003). Por ejemplo, es usual que las y los jóvenes piensen en no finalizar el último ciclo de educación diversificada, debido a que la pobreza les obliga a trabajar y contribuir con las entradas económicas de su familia. Por lo tanto, deciden insertarse en la vida laboral en un contexto donde las oportunidades se reducen al trabajo en las grandes plantaciones o la emigración (Pujol, 2006).

\section{b. INSTRUMENTO}

Para la recolección de los datos, se trabajó con el Cuestionario de Problemas Sociomorales (DIT-UCR por sus siglas en inglés) de Rest (1979), cuyos criterios de confiabilidad $y$ validez se determinaron tomando en cuenta diferentes análisis de dimensionalidad y consistencia interna. Se realizaron análisis de validez de constructo convergente y discriminante, análisis factorial de rotación Varimax y análisis de confiabilidad por coeficiente Alfa de Cronbach. Estos datos se obtuvieron a partir de los resultados de una prueba piloto $y$ del estudio principal (Monestel, 2011).

Previo a la prueba piloto se adaptó la versión corta traducida al castellano por Mestre, Perez, Samper y Frías (1999) del Defining Issues Test (DIT) de Rest (1979), para que pudiera ser accesible $y$ aplicable a la población rural escolarizada costarricense. Con este propósito se sometió a revisión lingüística y filológica. Además, siguiendo con las recomendaciones de Willis (2005) para el diseño y la validación de instrumentos, se realizaron entrevistas cognitivas con adolescentes del medio urbano y rural; es decir, se discutió la versión costarricense del instrumento con cuatro adolescentes 
(dos hombres $y$ dos mujeres) de Los Chiles $y$ Desamparados, con el fin de valorar la comprensión de los ítems y las historias que plantean un dilema moral (Monestel, 2011: 65).

Vale la pena mencionar que a través del DIT-UCR, han sido evidentes las estructuras de pensamiento moral preconvencional o esquema de intereses personales $y$ respeto unilateral de la autoridad, convencional o esquema de mantenimiento de las normas y postconvencional (Thoma, 2006). Este instrumento consta de tres dilemas sociomorales, los cuales deben ser evaluados de acuerdo con doce alternativas en una escala de cinco puntos. Cada historia enfatiza en la discusión de valores tales como la propiedad privada, la vida, la libertad de expresión y el bien común. De esta forma, se intenta indagar el razonamiento o estructuras de pensamiento moral, a través de los cuales las y los jóvenes resuelven los dilemas (Monestel, 2011: anexo 3).

El DIT-uCR es un instrumento complejo y se puede trabajar de varias maneras según sean los intereses de la investigación, de tal forma que se pueden abordar varios índices al mismo tiempo. En este estudio se calculó un índice global (D) de reflexión sociomoral, el cual pondera una puntuación calculada a partir de una escala. También se consideró un puntaje categórico de reflexión sociomoral que ubica a los y las participantes en un esquema de pensamiento moral predominante (preconvencinal, convencional o postconvencional), así como un índice $\mathrm{P}$, el cual pondera el puntaje de respuestas que tienen los sujetos en los ítems relacionados con el razonamiento postconvencional, midiendo hasta qué punto existe una reflexión que va más allá de la convención social. Finalmente, se realizaron análisis de pruebas $t$ para muestras independientes, con el fin de corroborar la influencia del contexto en las variables analizadas.

\section{RESULTADOS}

Antes de exponer los resultados, vale la pena destacar que durante el estudio piloto, se experimentaron dificultades en la comprensión de las instrucciones del DIT-uCR, sobre todo en el contexto rural, lo cual se vio reflejado en los resultados arrojados por dicha prueba con respecto a la consistencia interna del instrumento.

\section{a. VALIDEZ Y CONSISTENCIA INTERNA}

La literatura (Mestre et ál., 1999; Rest, Narvaez, Thoma y Bebeau, 2000; Eisenberg et ál., 2006) defiende que el DIT-UCR es sensible al ámbito educativo. Así, se pueden considerar las correlaciones entre la escolaridad y los índices del instrumento como criterios de validez convergente, a partir de la muestra del estudio principal. Los hallazgos comprueban los postulados teóricos acerca de las relaciones entre dicho criterio y la reflexión sociomoral, de tal forma que en ambos contextos se evidenciaron correlaciones positivas y significativas entre ambas variables, a saber: $r=.260^{*}$, $\mathrm{p}<0,05$ en Los Chiles y $r=.221^{*}, \mathrm{p}<0,05$ en Desamparados.

Asimismo, la validez discriminante se determinó comparando los tres índices del DITucr con los ítems de una Escala de Deseabilidad Social, la cual considera la tendencia acerca del grado en que las personas emiten respuestas socialmente deseables (Robinson, Schaver y Wrightman, 1991). Los resultados confirman que en ninguno de los casos existen correlaciones significativas entre los índices del DIT-UCR $y$ los ítems de dicha escala. Por lo tanto, se pueden hacer interpretaciones positivas acerca de la validez discriminante del DIT. En la Tabla 1 se muestran estos resultados (Monestel, 2011: anexo 6).

TABLA 1

CORRELACIONES CON LA ESCALA DESEABILIDAD

\begin{tabular}{ccc}
\hline & LOS CHILES & DESAMPARADOS \\
\hline DIT & & \\
D & .316 & .397 \\
P & .067 & .029 \\
Reflexión sociomoral categórica & .137 & .174 \\
\hline
\end{tabular}

Fuente: Elaboración propia.

* Correlación significativa al nivel 0,05** Correlación significativa al nivel 0,01 
Por otro lado, se realizó un análisis de consistencia interna para determinar la confiabilidad del instrumento. De acuerdo con esta prueba, los índices de Alfa de Cronbach fueron aceptables: $\alpha=.691$ para la zona rural y $\alpha=.725$ en la zona urbana. Por lo tanto, se puede decir que en el estudio principal, el instrumento presentó una adecuada consistencia interna en ambos contextos.

\section{b. COMPORTAMIENTO DE LAS VARIABLES}

A través del análisis de factores, se determinó el comportamiento de las variables que reflejan las formas predominantes de razonamiento moral. Este instrumento mostró que el agrupamiento de los ítems se comporta de la manera teóricamente esperada. De esta forma, dicho análisis partió del postulado que defiende que, si bien las personas tienden a una estructura de razonamiento predominante, esto no implica que dejen de utilizar los esquemas previamente alcanzados. Por lo tanto, el análisis factorial se basó en matrices de componentes factoriales que representaron los ítems de cada esquema, de acuerdo a lo estipulado en la teoría (Thoma, 2006). Los componentes que representan los postulados teóricos en las tres matrices factoriales explicaron más del $30 \%$ de la varianza del constructo, por lo que se puede decir que los ítems miden los esquemas preconvencional, convencional, postconvencional y sus fases de transición (Monestel, 2011: anexo 12).
Aunado a esto, los resultados del estudio principal revelan que la mayoría de adolescentes en ambos contextos se ubican predominatemente en el esquema convencional de reflexión sociomoral. De acuerdo al índice categórico calculado por el DIT, en el 85\% de los cuestionarios válidos de la zona rural, predomina dicha estructura de pensamiento, mientras que un $78 \%$ de los casos válidos en el medio urbano se revela una tendencia a la reflexión sociomoral convencional. Así, los hallazgos indican que predomina ampliamente el esquema convencional en la población adolescente costarricense escolarizada entre noveno y undécimo año.

En lo que respecta a las diferencias entre los contextos, la prueba $t$ para muestras independientes indicó que el esquema de reflexión sociomoral no se comporta significativamente diferente en ambas zonas $(t(180)=1.238, p$ $=.217$ ). Del mismo modo, de los indicadores ponderados del DIT-UCR, solo el índice que revela el pensamiento postconvencional (índice P) mostró diferencias significativas entre las muestras $(t(171)=-2.194, p=.030)$. Estos resultados sugieren que cuando se trata del esquema postconvencional medido por el DIT-UCR, el contexto parece tener alguna influencia en el comportamiento de la variable, de tal forma que la media de la zona rural mostró un índice más bajo que el de la urbana. En la Tabla 2 se muestran las estadísticas y los resultados de esta prueba para los índices ponderados (índice $\mathrm{D}$ o global e índice $\mathrm{P}$ o postconvencional).

TABLA 2

DIFERENCIAS DE LAS MEDIAS DE LOS ÍNDICES DEL DIT SEGÚN LA ZONA

\begin{tabular}{lcccccccc}
\hline & ZONA & $\mathrm{n}$ & MEDIA & $D E$ & MÍNIMO & MÁXIMO & $t$ & SIG. (BILATERAL) \\
\hline Índice P & Rural & 75 & 10,55 & 7,011 & 1 & 31 & &, 030 \\
& Urbana & 98 & 12,90 & 6,998 & 1 & 35 & $-.2,194$ &, 402 \\
\hline \multirow{2}{*}{ Índice D } & Rural & 93 & 13,03 & 1,623 & 19 & 33 & &,- 841 \\
& Urbana & 92 & 13,24 & 1,819 & 17 & 33 & & \\
\hline
\end{tabular}

Fuente: Elaboración propia. 


\section{DISCUSIÓN}

La falta de diferencias significativas en el comportamiento de las variables medidas por el DIT-UCR, se puede interpretar por el hecho de que las y los adolescentes de este estudio tengan en común estar inmersos en el sistema educativo formal. No obstante, es evidente que si bien la globalización ha transformando el medio rural con elementos presentes en regiones más urbanas, aún son evidentes diferencias significativas entre los contextos (Pujol, 2006; Pérez, 2001; De Grammont, 2008). De esta forma, el índice $P$ (moral postconvencional) parece ser la variable más sensible a tales diferencias $(t(171)=-2.194$, $p=.030$ ), demostrando un promedio más bajo de razonamiento moral postconvencional en Los Chiles (tabla 2).

Por otro lado, la naturaleza compleja del Cuestionario de Reflexión Sociomoral y las dificultades que se presentaron al trabajarlo en Los Chiles durante la prueba piloto, abren la discusión desde una perspectiva crítica de la psicometría, ya que se ha centrado en estudios que hacen de los ambientes urbanos una fuente de toda su generalidad, dejando al margen la ruralidad e invisibilizando sus necesidades particulares (Albuquerque, 2001). Partiendo de estos resultados, por ejemplo, se podría indagar sobre la equidad en el sistema educativo formal, ya que un instrumento que no presentó mayor problema para estudiantes urbanos, tuvo dificultades en su aplicabilidad en el contexto rural. A pesar de dichas dificultades, los resultados arrojan índices que evidencian aspectos de validez y consistencia interna, sobre todo en el estudio principal (Monestel, 2011).

Otro aspecto que interesa rescatar es que la teoría defiende que los esquemas maduros de reflexión sociomoral se vinculan positivamente con el nivel educativo, lo cual fue evidente a partir de los resultados que evidenciaron correlaciones positivas y significativas entre los índices del instrumento y la escolaridad (Eisenberg et ál., 2006; Mestre, Pérez, Samper y Frías, 1999; Rest et ál., 2000; Thoma, 2006). Este indicador no solo evidencia la validez del instrumento en ambos contextos, sino que además permite el análisis acerca del papel de la escuela en el desarrollo del pensamiento crítico de las $y$ los jóvenes inmersos en el sistema educativo formal, así como de su calidad con respecto a la formación de sujetos de derechos que ejerciendo la ciudadanía, a partir del análisis crítico de las convenciones sociales que los rigen, logren ser activos y activas en la transformación de su contexto.

En este punto, es importante considerar que podría mejorarse la calidad educativa ofrecida por el Estado, ya que se ha demostrado que existe una carencia de espacios reales de participación, en los cuales se estimule a las y los estudiantes a fomentar el desarrollo de su capacidad deliberativa. Por lo tanto, esta carencia podría limitar la reflexión sobre aquellos temas de orden público que les podría estar afectando (Castillo y Mora, 2009).

Es decir, el pasaje a la moral postconvencional podría ser estimulado por programas que promuevan la deliberación a través de espacios de participación (Castillo y Mora, 2009). Así, los resultados demostraron que el porcentaje de adolescentes que presentaron un esquema de pensamiento convencional fue predominante (85\% en Los Chiles y 78\% en Desamparados), mientras que el esquema postconvencional fue relativamente escaso, presentando promedios más bajos en la zona rural (tabla 2).

Ante estos indicadores, conviene rescatar el enfoque de la nueva ruralidad, argumentando que la exclusión social que ha sufrido dicho contexto como consecuencia de la globalización, se manifiesta en la falta de políticas públicas diseñadas para responder a las necesidades específicas de sus habitantes. Se puede evidenciar una deprivación educativa y cultural que se traduce en una mayor deserción escolar en la zona y una baja calidad educativa, la cual no responde a las nuevas exigencias impuestas por los cambios tecnológicos y del mercado laboral. Esta realidad reduce sus opciones de desarrollo y goce personal de la cultura, así como sus oportunidades laborales (Donas, 2001).

En este sentido, Los Chiles es un territorio caracterizado por ser un área rural marginal. Esta caracterización de la zona se plantea a partir de los bajos índices de desarrollo social, en donde los atributos agroecológicos son deficientes $y$ el acceso a mercados y centros de 
empleo es aislado, debido a que es un territorio ubicado en una zona fronteriza y representa un foco geográfico de pobreza (Janvry $y$ Sadoulet, 2004).

Este contexto de desventaja se ha atribuido a las estrategias socioeconómicas impulsadas desde la postguerra. Sin embargo, aún y cuando algunas tendencias intentaron mostrar la relación entre el fracaso de dichas fórmulas y los efectos de los proyectos de desarro1lo, aunados a sus prejuicios socioculturales, actualmente siguen predominando las políticas neoliberales que defienden una única receta de desarrollo válida para todos los países, cuyos principios se sustentan en la apertura económica, la no intervención pública y la perspectiva del análisis social coste-beneficio. Así, la evidencia histórica ha mostrado que el proceso de modernización aplicado durante la segunda mitad del siglo xx, en los países del Tercer Mundo, ha extendido la pobreza y la marginación social hasta límites sin precedentes (Trpin, 2005).

Ante esta realidad contextual, conviene rescatar la idea planteada por Turiel (2006), quien propone que existe una tendencia a que los esquemas de valores socialmente compartidos no corresponden con los grupos en desventaja. De esta forma, de acuerdo a estos postulados, se podría interpretar que las dimensiones cognitivas del desarrollo moral medidas por el DIT-UcR presentan índices más bajos en Los Chiles, ya que la situación real del grupo en estudio no corresponde con los esquemas de valores dominantes, los cuales se transmiten por la vía institucional en la que se inscribe el sistema educativo (Vygotsky, 1997).

Del mismo modo, se podrían relacionar estas diferencias con las historias planteadas en el instrumento, ya que hacen hincapié en valores asociados a circunstancias distintas a las que podrían presentarse en el contexto en estudio (Monestel, 2011: anexo 3). De esta forma, se entiende que el razonamiento moral es producto de la sociedad, su historia y sus estructuras formales (Thoma, 2006). De ahí que se caiga en la controversia de si la moral postconvencional planteada por Kohlberg, de la cual se derivan los enunciados del DIT-UCR, se relaciona más con criterios éticos de la cultura occidental hegemónica que con una lógica de pensamiento (Turiel, 2006; Thoma, 2006; Haskuka, Sunar y Alp, 2008).

La investigación del dominio moral cognitivo ha examinado las formas en que las ideologías culturales sirven para perpetuar prácticas discriminatorias que han tenido consecuencias negativas para ciertos grupos en desventaja social. Así, se propone que la autonomía, la equidad y la justicia, por ejemplo, representan criterios éticos contradictorios con los esquemas de valores de culturas colectivistas, en las cuales predominan las nociones del deber, la jerarquía y la interdependencia (Turiel, 2006 y Lapsley, 2006).

No obstante, los hallazgos del presente estudio concuerdan con la teoría (Turiel, 2006 $y$ Piaget, 1984) en la perspectiva de que durante la adolescencia, la cognición moral se basa en la necesidad de las normas establecidas por el sistema social tradicional para comprender las nociones sobre el bienestar común (esquema convencional de mantenimiento de las normas). Los juicios morales son entonces parte de las concepciones emergentes de los sistemas sociales, el respeto a las leyes y la autoridad institucionalizada. No es hasta el final de la adolescencia y la edad adulta que los juicios morales se lograran diferenciar de las reglas y las convenciones del sistema social (esquema postconvencional de moralidad de principios).

En síntesis, se podría hipotetizar que la falta de espacios para la discusión sobre la hostilidad del contexto, la falta de oportunidades $y$ la marginación de estos adolescentes, aunado a un sistema educativo que no promueve espacios para la deliberación (Castillo y Mora, 2009), podría relacionarse con las limitaciones en el pasaje de una reflexión moral convencional a una moral postconvencional. En este punto conviene rescatar la idea de que el conflicto es intrínseco a la condición humana o al menos, parte de la vida cotidiana en las sociedades. Además de la armonía común y social, hay desacuerdos y falta de armonía dentro de las culturas, así como hay tradición, existe también subversión (Turiel, 2006). 
Una vez más, se retoma la perspectiva según la cual la evolución de la moral común se relaciona con el debate y la negociación colectiva, de tal forma que logra permanencia al obtener el apoyo general de la comunidad. Este último punto es importante ya que trae a colación la noción del relativismo moral, el cual representa una preocupación para los investigadores del juicio moral (Thoma, 2006 y Turiel, 2006).

La noción de la moral común es una respuesta a dicho relativismo, ya que describe el desarrollo de procesos de deliberación y sus sistemas de aplicación en la comunidad. De esta forma, esta perspectiva cuestiona la universalidad moral tal y como la entendía Kohlberg (1992) y plantea una pregunta empírica acerca de los procesos de pensamiento que trascienden las concepciones morales a través de las culturas, sin perder de vista los procesos psicosociales en la definición de lo moral (Thoma, 2006).

Sin embargo, esta pregunta empírica todavía está lejos de ofrecer respuestas definitivas sobre el papel de la praxis en el desarrollo cognitivo (Turiel, 2006). Así, entendiendo el fenómeno moral como un producto social, construido a partir de una historia y de estructuras sociales que determinan y se determinan a partir de la actividad humana, se puede decir que si bien podría existir un funcionamiento común, lo cierto es que el orden social aún plantea temas morales contradictorios que no han sido resueltos (Turiel, 2006).

Por consiguiente, es importante considerar que el funcionamiento moral probablemente adquiera un rumbo distinto según sean las condiciones en las que se desarrollen las personas, ya que las injusticias sociales, la oposición, la resistencia y los actos de subversión que prevalecen en el mundo, a menudo plantean problemas que requieren una gran cantidad de trabajo emocional y conceptual, lo cual da cuenta del conflicto social (Turiel, 2006).

Finalmente, es de esperar que las y los adolescentes del grupo rural muestren diferencias en su pasaje a la reflexión sociomoral postconvencional. Estos hallazgos se pueden explicar a partir de las circunstancias sociales en las que se encuentran estos jóvenes y su posicionamiento frente a un sistema de normas $y$ valores que los coloca en desventaja. Este argumento aunado al cuestionamiento del DirUCR como instrumento que priva la medición de una moral particular, podría dar alguna luz sobre los resultados de esta investigación. Por ende, queda abierto el debate sobre la pregunta empírica que plantea la universalidad del funcionamiento moral.

\section{REFERENCIAS}

Acosta, Irma. "El enfoque de la nueva ruralidad como eje de las políticas públicas. ¿Qué podemos esperar?". Revista Electrónica Zacateca sobre Población y Sociedad 32 (3). 2008: 1-20.

Aguilar, M. E.; Angulo, L.; Cerdas, Y.; Céspedes, E.; Monge, M. E.; Ovares, S. y Van Kampen, P. Un acercamiento a la educación general básica de seis países centroamericanos. Heredia: Fundación UNa, 2003.

Albuquerque, José Batista. "Aproximación metodológica desde la Psicología Social a la investigación en zonas rurales". Estudios Agrosociales y Pesqueros 191. 2001: 225-233.

Alvarado, Sara Victoria; Ospina-Alvarado, María Camila y García, Claudia María. "La subjetividad política y la socialización política, desde las márgenes de la Psicología Política". Revista Latinoamericana de Ciencias Sociales, Niñez y Juventud 10 (1). 2012: 235-256.

Blasi, Augusto. "Brinding moral cognition and moral action: a critical review of the literature". Psychologycal Bulletin 88 (1). 1990: 1-45.

Blasi, Augusto. "Emotions and moral motivation". Journal for the Theory of Social Behavior 29 (1). 1999: 5-19.

Brislin, Richard. "The wording and translation of research instruments". Field methods in cross-cultural research. Walter Lonner y John Berry (eds.). Beverly Hills. Sage, 1986: 137-164.

Canales, Alejandro; Martínez, Jorge; Reboiras, Leandro y Rivera, Felipe. Migración y salud en zonas fronterizas: informe comparativo sobre cinco fronteras seleccionadas. Santiago: CELADE/CEPAL, 2010. 
Carmiol, Ana María y Cenko, Enila. "Weaving sociocultural change and cognitive development together". Culture \& Psychology 13 (4). Sage Publications, 2007: 500-512.

Carrión, Fernando. "Las nuevas tendencias de la urbanización en América Latina". La Ciudad construida. urbanismo en América Latina. Fernando Carrión (ed.). Quito: FLACso, 2001: 7-24.

Castillo, Andrés y Mora, Carolina. "Estrategia pedagógica para la deliberación y el razonamiento sociomoral en jóvenes de secundaria”. Actualidades en Psicología 23-24. 2009: 103-129.

Castro, Ricardo. "Estilos y figuras de apego predominantes en la adolescencia: estimación de la validez y la coherencia interna del "Cuestionario de estilos de apego" en el contexto costarricense". [Tesis de Licenciatura en Psicología]. Universidad de Costa Rica, 2006.

Clark-Carter, David. Quantitative psychological research: a student's handbook. New York: Psychology Press, 2004.

Cohen, Louis y Manion, Lawrence. Métodos de investigación educativa. España: La Muralla, 1990.

Cole, Michael. Psicología cultural. Una disciplina del pasado y del futuro. Madrid: Morata, 1999.

Cole, Michael y Yrjö, Engeström. "A culturalhistorical approach to distributed cognition". Distributed cognitions: psychological and educational considerations. G. Salomon (ed.). New York: Cambridge University Press, 1993: 1-46.

Comunian, Anna y Gielen, Uwe. "Moral reasoning and prosocial action in Italian culture". The Journal of Social Psychology 135 (6). 1995: 699-706.

Cortina, Adela. "Ética, ciudadanía y modernidad". CyberHumanitas. 2003: 1-13. En: <http://scholar. googleusercontent.com/scholar?q=c ache:TLBXzYz8Fs0s:scholar.google.com/+A + cortina+etica+ciudadania\&hl=en\& as_sdt $=0,10>$ [consultado 20 de abril de 2013].
Cortina, Adela. "Ciudadanía intercultural". Philosophica 27. 2006: 7-15.

Davis, Mark. "Measuring individual differences in empathy: evidence for a multidimensional approach". Journal of Personality and Social Psychology 44 (1). 1983: 113-126.

De Grammont, Hubert. "El concepto de nueva ruralidad". La nueva ruralidad en América Latina. Avances teóricos y evidencias empíricas. Edelmira Pérez, María Farah y Hubert De Grammont (eds.). Bogotá. Pontificia, 2008: 23-44.

Díaz-Palacios, José Alfredo. "Calidad educativa: un análisis sobre la acomodación de los sistemas de gestión de la calidad empresarial a la valoración en educación". Tendencias Pedagógicas 21. 2013: 177-194.

Diez Hurtado, Alejandro. "Organizaciones e integración en el campo peruano después de las políticas neoliberales". ¿Una nueva ruralidad en América Latina? Norma Giarracca (ed.). Buenos Aires. clacso, 2001: 191-220.

Donas, Solum. "Adolescencia y juventud: viejos y nuevos desafíos en los albores del nuevo milenio". Solum Donas (ed.). Cartago. Libro Universitario Regional (EULAC-GTZ), 2001: 41-56.

Echeverri, Rafael y Ribero, María del Pilar. Nueva ruralidad: visión del territorio en América Latina y el Caribe. San José: Instituto Interamericano de Cooperación para la Agricultura, 2002.

Eisenberg, Nancy; Spinrad, Tracy y Sadovsky, Adrienne. "Empathy-related responding in children". Handbook of Moral Development. Melanie Killen y Judith Smetana (eds.). New Jersey. Lawrence Erlbaum Associates, 2006: 7-35.

Fogel, Ramón. "La estructura y la coyuntura en las luchas del movimiento campesino paraguayo". ¿Una nueva ruralidad en América Latina? Norma Giarracca (ed.). Buenos Aires. clacso, 2001: 221-242.

Gibbs, John; Basinger, Karen y Fuller, Dick. Moral maturity. Measuring the development of sociomoral reflection. New Jesey: Erlbaum,1992. 
Haskuka, Mytaher; Sunar, Diane y Alp, Ercan."Warexposure, attachment style and moral reasoning". Journal of Cross Cultural Psychology 39 (4). 2008: 381401.

Instituto Interamericano de Cooperación para la Agricultura (IICA). Desarrollo Rural Sostenible enfoque territorial: la experiencia del IICA en Brasil. Brasilia: IICA, 2002.

Instituto Interamericano de Cooperación para la Agricultura (IICA). Los Chiles, Upala, Guatuso y La Cruz: dinámicas territoriales en la zona norte de Costa Rica. Carlos Granados, Alonso Brenes, Luis Cubero, Heiner Murillo y José Carlos Arce (coords.). San José: ıIca, 2007.

Instituto Interamericano de Cooperación para la Agricultura (IICA). Informe anual 2008. Contribución del IICA a la agricultura y el desarrollo de las comunidades rurales en Costa Rica. San José: IICA, 2009.

Instituto Interamericano de Derechos Humanos (IIDH). Propuesta curricular y metodológica para la incorporación de la educación en derechos humanos en la educación formal de niños y niñas entre 10 y 14 años de edad. San José: IIDH, 2006.

Instituto Nacional de Estadística y Censos (INEC). Conceptos básicos. San José: inec, 2004. En: <http://www.inec.go.cr/> [consultado el 30 de abril de 2008].

Instituto Nacional de Estadística y Censos (INEC). Anuario estadístico 2007. San José: INEc, 2007. En: <http://www.inec.go.cr/> [consultado el 25 de agosto de 2009].

Janvry, Alain y Sadoulet, Elisabeth. "Hacia un enfoque territorial del desarrollo rural". Ponencia presentada en el Cuarto Foro Temático Regional de América Latina y el Caribe: ¿Cómo cosechar las oportunidades disponibles?: el desarrollo rural en el siglo 21. San José, Costa Rica. Octubre 2004.

Kay, Cristóbal. "Algunas reflexiones sobre los estudios rurales en América Latina". Íconos. Revista de Ciencias Sociales 29. 2007: 31-50.
Kohlberg, Lawrence. Psicología del desarrollo moral. Bilbao: Desclée de Brouwer, 1992.

Krauscopf, Dina (1982). Adolescencia y educación. $13^{\mathrm{a}}$ edición. San José: Editorial Universidad Estatal a Distancia, 2007.

Lapsley, Daniel K. "Moral Stage Theory". Handbook of Moral Development. Mellanie Killen y Judith Smetana (eds.). New Jersey: Lawrence Erlbaum Associates, 2006: 7-35.

Llambí, Luis y Pérez, Edelmira. "Nuevas ruralidades y viejos campesinismos. Agenda para una nueva sociología rural latinoamericana". Cuadernos de Desarrollo Rural 4 (59). 2007: 37-61.

Margulis, Mario. "Juventud: una aproximación conceptual". Adolescencia y juventud en América Latina. Solum Donas (ed.). Cartago. Libro Universitario Regional (EULAC-GTZ), 2001: 41-56.

Mestre, María Vicenta; Pérez, Esteban; Samper, Paula y Frías, María Dolores. "Instrumentos de evaluación del razonamiento moral". Psicología moral y crecimiento personal. María Vicenta Mestre y Esteban Pérez (eds.). Barcelona: Ariel, 1999.

Mestre, María Vicenta; Frías, María Dolores y Samper, Paula. "La medida de la empatía: análisis del Interpersonal Reactivity Index". Psicothema 16 (2). 2004: 255-260.

Miranda, Carlos y Matos, Aureliano. Desarrollo rural sostenible enfoque territorial: la experiencia del IICA en Brasil. Brasilia: IICA, 2002.

Monestel, Natacha. "Desarrollo sociomoral $y$ estilos de apego en adolescentes del cantón de Los Chiles". [Tesis de Licenciatura]. Universidad de Costa Rica, 2011.

Pérez, Edelmira. "Hacia una nueva visión de lo rural". ¿Una nueva ruralidad en América Latina? Norma Giarracca (ed.). Buenos Aires: cLacso, 2001: 17-30.

Piaget, Jean (1932). El criterio moral en el niño. Barcelona: Martínez Roca, 1984.

Piaget, Jean (1964). Seis estudios de Psicología. Barcelona: Labor, 1991. 
Piñeiro, Diego. "Población y trabajadores rurales en el contexto de transformaciones agrarias". ¿Una nueva ruralidad en América Latina? Norma Giarracca (ed.). Buenos Aires. clacso, 2001: 269-288.

Portilla, Melania y Barrantes, Celia. Juventud Rural y Desarrollo Sostenible. Instituto Interamericano de Cooperación para la Agricultura, 2003. En: <http://www. iica.int/Esp/Programas/Territorios/ Documentos\%20Desarrollo\%20Rural\%20 Sostenible/sinopsis/S.3.2003.pdf>

Programa Estado de la Nación. "El mundo rural en transición”. III Informe Estado de la Nación. 1997. En: <http://www. estadonacion.or.cr/Info1997/paginas/ cap1-96e.htm> [consultado el 30 de abril de 2008].

Pujol, Rosendo. "Diferenciales entre zonas urbanas y rurales de Costa Rica: análisis estadístico de la información del censo del 2000". Simposio Costa Rica a la Luz del Censo. San José, 2006. En: <http:// ccp.ucr.ac.cr/noticias/simposio/ponencia/ ponencia.htm > [consultado el 20 de julio de 2008].

Ratner, Carl. "Three approaches to cultural psychology: a critique". Cultural Dynamics 11 (1). 1999: 7-31.

Reguillo, Rossna (1991). Las bandas: identidad urbana y usos de la comunicación. Jalisco: IтEso, 1995.

Rest, James. Development in Juding Moral Issues. Minneapolis: University of Minnesota, 1979.

Rest, James; Narvaez, Darcia; Thoma, Stephan J. y Bebeau, Muriel. "A Neo-Kohlbergian approach to morality research". Journal of Moral Education 29 (4). 2000: 381-395.

Robinson, John; Schaver, Phillip y Wrightman, Lawrence. Measures of personality and social psychologia lattitudes. London: Academic Press, 1991.

Rodríguez, José Miguel. "Gramática de la moral: la adjetivación de las acciones de los Bribris". [Tesis de Licenciatura en Psicología]. Universidad de Costa Rica, 2000.
Rushton, Philippe; Chrisjohn, Roland y Fekken, Cynthia. "The altruistic personality and the Self-Report Altruism Scale". Personality \& Individual Differences 2. 1981: 293-302.

Sanabria, Jorge. Reflexiones en torno a la niñez y adolescencia en riesgo social en la vía pública. San José: unicef, 2002.

Sepúlveda, Sergio y Zúñiga, Hannia. "Elementos conceptuales del desarrollo rural sostenible". Gestión del desarrollo sostenible en territoriales rurales: métodos para la planificación. Sergio Sepúlveda (ed.). San José. IICA, 2008: 1-132.

Solórzano, William. "Poblamiento y colonización de la región norte de Costa Rica". Trayectorias y disyuntivas del Agro en la Zona Norte de Costa Rica. Mario Samper (ed.). San José. Instituto de Investigaciones Sociales, UCR. 2005: 11-32.

Tapia, Napoleón; Castro, Ricardo y Monestel, Natacha. "El desarrollo sociomoral de adolescentes de Costa Rica según el modelo de Gibbs". Revista Latinoamericana de Psicología 39 (3). 2007: 449-471.

Tappan, Mark. "Moral functioning as mediated action". Journal of Moral Education 35 (1). 2006: 1-18.

Teubal, Miguel. "Globalización y nueva ruralidad en América Latina”. ¿Una nueva ruralidad en América Latina? Norma Giarracca (ed.). Buenos Aires. CLACSO, 2001: 17-30.

Thoma, Stephen. "Research on the Defining Issues Test". Handbook of Moral Development. Mellanie Killen y Judith Smetana (eds.). New Jersey. Lawrence Erlbaum Associates, 2006: 67-91.

Trpin, Verónica. "El desarrollo rural ante la nueva ruralidad. Algunos aportes desde los métodos cualitativos". Revista de Antropología Iberoamericana 42. 2005: $1-15$.

Turiel, Elliot. "Thought, emotions, and social interactional processes in moral development". Handbook of Moral 
Development. Mellanie Killen y Judith Smetana (eds.). New Jersey. Lawrence Erlbaum Associates, 2006: 7-35.

Valsiner, Jaan. Child development in cultural context. Toronto: Hogrefe and Huber, 1989.

Vygotsky, Lev Semiónovich (1926). Educational Psychology. Boca Raton, fL.: St. Lucie Press, 1997.
Willis, Gordon. Cognitive interviewing: a tool for improving questionnaire design. London: SAGE. 2005.

Wertsch, James. Vygotsky y la formación social de la mente. Barcelona: Paidós, 1988.

Fecha de ingreso: 15/02/2012 Fecha de aprobación: 01/05/2013 
